\title{
XII. Ueber den Aragonit von Sicilien und seine Structur.
}

\author{
Von \\ C. Viola in Rom.
}

(Hierzu Tafel IV.)

In den verschiedenen Schwefellagern von Sicilien, besonders in denjenigen von Racalmuto, Cianciana und Girgenti, kommen oft in den tieferen Ablagerungen der Schwefelformation die bekannten grossen Aragonitkrystalle, verwachsen mit Cölestin und mit schönen Schwefelkrystallen, vor. Da ich einige derselben erhielt, und die schöne Sammlung solcher Krystalle der hiesigen geologischen Anstalt mir zur Verfugung stand, babe ich einige Versuche zur Bestimmung der Symmetrie des Aragonits angestellt.

Der Aragonit erscheint in schönen, weissen oder bläulichen, oft sehr klaren Krystallen von hexagonalem Aussehen. Es sind nur von der Basis und den FJächen des primären Prismas und höchstens noch von $\{010\}$ und $\{0 T 0\}$ begrenzte $Z$ willingskrystalle des gewöhnlichen Gesetzes, fast immer Durchdringungszwillinge mit ganz beliebiger Verwachsungsfläche. Zwei Zwillinge allein bringen schon das hexagonale Ausseben des Aragonitkrystalles hervor, wie die Fig. 1, Taf. IV schematisch zeigt; doch eine so einfache Combination kommt selten vor, da sehr oft noch ein drittes Individuum sich an einen der Zwillinge mil der gemeinscbaftlichen Fläche (1T0) anlehnt, dann aber auch ein viertes, sich an den zweiten Zwilling anschliessendes Individuum binzutritt, ohne die schon von den zwei $Z$ willingen bestimmte hexagonale Form zu beeinflussen. Die Fig. 2 stellt $z$ wischen gekreuzten Nicols einen zu (001) parallelen Schnitt eines solchen Aragonitkrystalles dar, und die Fig. 3 giebt schematisch ein Beispiel einer solchen Combination wieder.

Auf der Basisfläche eines Zwillingskrystalles beobachtet man Eigenthumlichkeiten, die einiges Interesse erwecken. Vorerst ist zu bemerken, dass streng genommen keine eigentliche Basis vorhanden ist, sondern nur eine grosse Anzahl von Vicinalflächen der Fläche (001), die zur Kante [100] 
parallel sind, so dass auf den er'sten Blick die Basis selbst von zur Brachydiagonale parallelen Streifen durchzogen erscheint. Die Fig. 4 stellt die obere Ansicht desjenigen Aragonitkrystalles dar, von dem wir den Schnitt Fig. 2 genommen haben. Durch Vergleichung dieser zwei Bilder ersieht man den Zusammenhang zwischen Verwachsungsfläche und Begrenzung der Streifung auf der Basis von Zwillingen. Die von den Vicinalflächen herkommende und zur Brachydiagonale parallele Streifung grenzt nicht scharf an der Verwachsungsebene von zwei Individuen ab. Die im Schnitte erscheinende Verwachsungslinie ist vielmehr zahnartig in der Weise, dass die Zähne parallel zur gemeinschaftlichen Fläche (110) übergreifen, während die Abgrenzung der Streifungen treppenartig und beziehungsweise parallel zur Brachydiagonalen ist. Mit anderen Worten: die Streifung eines Individuums ragt ohne Unterbrechung in das andere hinein. Diese Verhältnisse erscheinen deutlich, wenn man einen Dünnschliff in der Nähe der Basis (001) zur Verfügung bat, denn im durchfallenden polarisirten Lichte nimmt man die Grenzen der Zwillinge und bei einfallendem Lichte die Streifungen an der oberen Seite der Basis wahr. Dies ist schematisch in der Fig, 5 dargestellt, wo die Streifungen mit ganzen und die Zwillingsubergreifungen mit unterbrochenen Linien angegeben sind.

Die in den Figuren 2 und 4 zum Vorschein kommenden Spaltungen gehen durch kleine, in ein Individuum eingedrungene Theile des anderen. Sie stellen offenbar die weniger widerstandsfähigen Stellen des Krystalles dar.

Rund um die hexagonale Peripherie eines Zwillingskrystalles herum sieht man in der Nähe der Basisfäche kleine Einschnitte (s. Figg. 2 und 4), die, obwohl nicht scharf abgegrenzt, doch auf die Flächen (111) und (021) von einem oder gleichzeitig von zwei Individuen hindeuten.

Um festzustellen, in welcher Klasse der Aragonit krystallisirt, resp. was fur eine Structur er hat, habe ich Aetzfiguren zu Hulfe genommen. Bekanntlich hat Hr. Beckonkamp I) sowohl auf der Basis (001), als auch auf den Flächen des Grundprismas sehr schöne Aetzfiguren erhalten, die neben den Erscheinungen der Pyroëlektricität ihm dazu dienten, die Aragonitkrystalle als Zwillinge von niederer Symmetrie zu erkennen. Ich babe durch stark verdunnte Salzsäure auf der Basis Aetzfiguren herzustellen versucht und erhalten.

Die Fig. 6 zeigt schematisch solche durch Salzsäure auf der Basisfäche erhaltene Aetzfiguren auf der Grenze zweier Zwillinge. Die Salzsäure lässt die Streifung auf der Basis schnell und vollkommen verschwinden, und an deren Stelle treten langgestreckte spindelförmige Aetzfiguren, deren Symmetrieebenen parallel zur Brachydiagonale verlaufen. Ich habe bei den

1) Diese Zeitschr. 1888, 14, 375. S. auch 19, 241; 20, 261. 
Versuchen mehr oder weniger stark verdünnte Salzsäure angewendet, und dabei doch immer Aetzfiguren von derselben Form, aber von verschiedener Grösse erbalten. Die Seiten dieser Aetzspindeln bilden ungefähr einen Winkel von $110^{\circ}$ bis $115^{\circ}$ mit einander, so dass sie wohl den Flächen des Prismas $\{120\}$ entsprechen mögen. Es ist nicht genau bestimmt, ob diese Aetzfiguren auch Symmetrieebenen besitzen, welche parallel zu $\{100\}$ wären.

Von grösserer Wichtigkeit noch sind die naturlichen Aetzfiguren des Aragonits, welche auf den Flächen des Prismas $\{110\}$ bei den sicilianischen Krystallen beobachtet werden.

Die naturlichen Aetzfiguren des Aragonits werden durch ein sehr gunstiges Lösungsmittel hervorgerufen, nämlich durch Kohlensäure.

Schon von Lasaul ${ }^{1}$ ) hatle, indem er die Pseudomorphosen des Calcits nach Aragonil erklärle, beobachtet, wie die Kohlensäure die Aragonitsubstanz auflöst, durch das Wasser dieselbe fortgefubrt wird, und in den dadurch entstandenen Vertiefungen kohlensaurer Kalk sich in Form von Calcit niederschlăgt. Es ist wichtig, von Lasaulx's eigene Worte hier wiederzugeben:

"Wird nun der Aragonit aufgelöst und fortgeführt, so entstehen hohle Skelette, die sich successive mit Calcit erfüllen, und endlich ist dann der ganze Aragonitkrystall in ein Aggregat von Calcitskalenoëdern verwandelt.

An dem Aragonitkerne zeigt sich, dass seine Auflösung ganz regelmässig erfolgt, der Aragonit zerfällt in lauter einzelne Längsfasern, und die leeren Zwischenrăume erfüllen sich mit Calcit.

"Längs der Grenzen der einzelnen im Aragonitzwilling vereinigten Individuen erfolgt die Fortfübrung der Substanz am schnellsten.

"Ein solcher Aragonitkrystall zeigt dann im Inneren ein Gerüst von noch erhaltenen $\Lambda$ ragonitbalken und -wänden, durch die den ursprünglichen $\mathrm{Zwillingsgrenzen}$ entsprechende Hohlräume getrennt. "

Zur Entscheidung, ob solche Aggregate von Calcit nach Aragonit als Paramorphosen oder Pseudomorphosen aufzufassen sind, macht von Lasaulx folgende, noch heute geltende Bemerkungen:

"Die Betrachtung der verschiedenen Stadien in der Bildung dieser Pseudomorphosen ergiebt, dass alle Erscheinungen sehr wohl ihre Erklärung finden, wenn man hier lediglich die Vorgänge einer mechanischen Ausfüllungs- und Umhüllungspseudomorphose annimmta. "An Stelle des fortgeführten Aragonits tritt Calcit, jedoch erst, weon der freie haum geschaffen, der ihn aufnimmt a... .

nWo also ein Mineral durch blosse Auflösung forlgeführl, und nun in seiner Hohlform ein mit jenem polymorphes Mineral nur durch Umhüllung und Erfüllung abgelagert wird, da liegt natürlich keine Paramorphose vor ", etc.

Es ist interessant, die durch die Kohlensäure entstandenen, v on Lasaulx erwähnten $Z$ wischenraume ihrer Bildung nach zu verfolgen, was man auf irgend einer Fläche des Prismas $\{110\}$ thun kann.

1) Beobachtungen in den Schwefeldistricten von Sicilien. N. Jahrb. f. Min. etc. 1879, 490. Ref. in dieser Zeitschr. 5, 389. 
Aus der Grösse der verschiedenen Vertiefungen kann man schliessen, dass die naturliche Aetzung in der Mitte der Fläche (110) beginnt und gegen die Peripherie sich ausdehnt.

Die Vertiefungen an der Peripherie haben die Form von Pyramiden mit gleichschenklig dreieckiger Basis, während gegen die Mitte der Fläche (110) die einzelnen Gruben so in einander verschmelzen, dass dadurch Aelzhtigel zurückbleiben. Die Fig. 7 ist eine getreue Wiedergabe solcher Aetzhtugel und Aetzvertiefungen auf der Fläche (110), und schief gesehen auch auf der Fläche (1T0). Die Fig. 8 stellt dagegen diese Aetzfiguren schematisch dar. Es ist wichtig, diese Aetzhúgel speciell zu untersuchen; jeder derselben besteht aus der Fläche (110) des Krystalles und aus drei schiefen Flächen, die auf (1.10) drei Kanten bestimmen; zwei derselben sind mit Bezug auf [001] symmetrisch, wäbrend die dritte Kante mit [001] parallel ist.

Die durch diese letztere Kante gehende schiefe Fläche des Aetzhugels ist zu der Fläche (1T0) parallel. Der Winkel, welchen die anderen zwei Kanten einschliessen, lässt sich auf dem Mikroskoptische auf ca. $63^{0}$ messen. Die Winkel der zwei geneigten Flachen mit (110) betragen ungefähr $53^{\circ}$, ein nach verschiedenen Messungen berechnetes Mittel.

Wenn diese zwei Flächen mit den Flächen (231) und (23T) des Aragonits identificirt werden, wie in der Fig. 9 angegeben ist, so erhält man fur die Axenverhältnisse des Aragonits:

$$
a: b: c=0,608: 1: 0,720 \text {. }
$$

Das Gleiche, was fur die Fläche $(110)$ angegeben ist, gilt auch für die Fläche (1T0). Auch hier bestehen die Aetzhugel aus den Flächen (1T0),

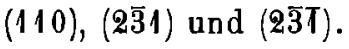

Auch aus den naturlichen, wie aus den von Beckenkamp kústlich erhaltenen, ähnlichen Aetzfiguren auf den Flächen des Prismas $\{110\}$, ist offenbar zu schliessen, dass wenigstens eine Symmetrieebene und wenigstens eine Symmetrieaxe vorbanden ist.

Alle weissen, nicht ganz durchsichtigen Krystalle der sicilianischen Ablagerungen baben solche soeben beschriebene Aetzhugel und Aetzhohlräume, die nach und nach mit Calcit- und Schwefel-Krystallen, wie von L a s a ulx selbst schon bemerkte, ausgefullt werden.

Die durchsichtigen, bläulichen Aragonitkrystalle hingegen zeigen auf den Flächen des Prismas $\{110\}$ andere Aetzfiguren. Diese letzteren sind zugespitzt gegen die Basis und symmetrisch mit Bezug auf die Kante [001], wie in der Fig. 10 schematisch dargestellt ist.

Um einen annehmbaren Schluss aus diesen Beobachtungen zu ziehen, müssen wir die Structur des Calcits zu Hulfe nehmen, welcher ein geringeres specifisches Gewicht hat als der Aragonit. Und da angenommen werden darf, dass die Molekeln des Calcits und des Aragonits gleich 
seien ${ }^{1}$, so muss die Structur des Aragonits aus derjenigen des Calcits durch Polymerisation entstehen.

Der Calcit krystallisirt in der skalenoëdrischen Symmetrie, welcher sechs verschiedene Structuren angehören. Wegen der gleichwerthigen Spaltungen des Calcits nach den Flächen des Grundrhomboëders kann die Structur des Calcits keine andere sein als $\mathfrak{D}_{3, d}^{6}$ (Bezeichnung von Schönflies) gleich $47 h$ (Fedorow'sche Numerirung).

Diese Structur ist in Fig. 11 schematisch dargestellt. Jeder Molekelhaufen besteht aus zwölf Molekeln, von denen je ein Paar symmetrisch in Bezug auf eine Symmetrieebene ist, und sechs Molekelpaare stehen in Bezug auf eine sechszählige Inversionsaxe symmetrisch. Daher hat jeder Molekelbaufen folgende Symmetrieelemente : eine sechszählige Inversionsaxe, drei durch diese Axe gehende Symmetrieebenen und drei zweizäblige auf letztere senkrechte Symmetrieaxen. Die betreffenden Translationen sind parallel zu den drei Kanten des Grund- oder Spaltungsrhomboëders.

Um diese Structur besser zu veranschaulichen, habe ich drei parallele aufeinanderfolgende Schichten von Molekelhaufen in der Weise dargestellt, dass die Molekeln der obersten Schicht am stärksten, diejenigen der zweiten etwas schwächer und endlich diejenigen der unlersten ganz schwach ausgezogen sind.

Die Molekeln selbst sind bald voll ausgezogen, bald leer, je nachdem sie auf der unteren oder auf der oberen Seite der in Betracht kommenden Schicht gelegen sind. Die zweizähligen Symmetrieaxen sind durch unterbrochene Linien dargestellt, und zwar stärker und schwächer je nach der Lage derselben in den verschiedenen Schichten.

Die verticalen sechszähligen Inversions- und gleichzeitig dreizähligen Symmetrieaxen sind auf dem Papier durch kleine, dreiseitige, vollausgezogene Dreiecke angegeben.

Die verticalen Symmetrieebenen sind durch ganz ausgezogene Linien dargestellt.

Die in der Mitte zwischen je drei sechszähligen Inversionsaxen vorhandenen, dreizähligen, rechtsdrehenden Schraubenaxen sind in der Zeichnung nicht aufgetragen.

Durch diese Art und Weise der Bezeichnung giebt die Fig. 11 ein getreues und klares Bild der Structur $\mathfrak{D}_{3, d}^{6}$ des Calcits, das dem Verständnisse der gedachten Structur von Aragonit dienen soll.

Wir gehen jetzt dazu uber, zu untersuchen, welche möglichen Structuren aus derjenigen des Calcits mit Hülfe der Polymerisation entstehen können.

Wenn je zwei Molekeln des Calcits, nämlich zwei voll und ebenso

1) G. Wulff, diese Zeitschr. 24, 509. 
zwei leer in der Zeichnung angegebene Molekeln sich miteinander vereinigen, so verschwinden sämmtliche verticale Symmetrieebenen, und die daraus entstehende Structur ist $\mathfrak{E}_{3, i}^{2}$ (Schoenflies), 52 $s$ (Fedorow), welche in die rhomboëdrische Symmetrie gehört. Man erhält also die Structur des im Dolomit enthaltenen kohlensauren Kalks. Wenn weiter in der Structur $\mathfrak{6}_{3, i}^{2}$ alle Molekeln sich vereinigen, so verschwindet jede Symmetrie, und es entsteht die einfachst mögliche Homogenität, welche zur asymmetrischen Klasse gehört.

Wir können uns ferner noch eine in Bezug auf die sechszählige Inversionsaxe nicht symmelrische Polymerie denken. Stellen wir uns nămlich vor, dass alle Molekelhaufen eine Verdichtung nach einer bestimmten Richtung erfabren. Dies ist in der Fig. 12 dargestellt, wobei, ebenso wie vorher zum besseren Verständniss der Structur, drei horizontale, ubereinander gelegene Schichten von Molekelhaufen in die Zeichnung eingetragen sind. Wir sehen daraus, dass jeder Molekelhaufen mit Bezug auf eine zweizählige Symmetrieaxe und eine darauf senkrechte Symmetrieebene symmetrisch ist, d. h. wir erhalten eine Structur, welche zur prismatischen Klasse (Groth) gehört.

Ferner können je vier Molekeln auf jeder Seite der Symmelrieebene sich vereinigen, und man erhält eine zur domatischen Klasse (Groth) gehörige Structur. Endlich können die Molekeln so vereinigt gedacht werden, dass die Symmetrieebenen verschwinden, und daraus die zur sphenoidischen Klasse (Groth) gehörige Structur entsteht.

Die aus der Structur $\mathfrak{D}_{3, d}^{6}$ des Calcits sich herausbildenden möglichen Structuren sind daher $\mathfrak{E}_{3, i}^{2}\left(5_{2}^{2} s\right), \mathfrak{E}_{2, h}^{3}(8 s), \mathfrak{\mho}_{s}^{3}(6 s), \mathfrak{G}_{2}^{3}(4 s)$ und $\mathfrak{E}_{1}(1 s)$, deren erste der rhomboëdrischen Klasse, die drei folgenden dem monoklinen und die letzte dem triklinen Systeme angehören.

Ich habe hier die Symbole von Sch oe nflies und in Klammern die Fedorow'sche Nummerirung angewendet.

Durch diese theoretischen Betrachtungen ist die Krystallisation des Aragonits innerhalb füf Structuren begrenzt. Um darin die richtige Wahl zu treffen, haben wir noch die vorher beschriebenen Versuche zu Hulfe zu nehmen. Da nun die natürlichen Aetzfiguren des Aragonits sowobl auf Symmetrieebenen als auch auf Symmetrieaxen hindeuten, so liegt auf der Hand, dass die Structur des Aragonits nur $\mathfrak{\Xi}_{2, h}^{3}$ sein kann, welche zur prismatischen Klasse (monoklin holoëdrisch) gebört.

Wir haben nun für den Aragonit dieselbe Erscheinung wie bei anderen polymorphen Substanzen, nämlich, dass die Molekelhaufen die prismatische Symmetrie besitzen, während das Raumgitter dem rhombischen Systeme entspricht. Wenn wir nun unter Pseudomorphismus einen viel weiteren Begriff verstehen wollen, als es gewöhnlich geschieht, so können wir uns, was den Aragonit anbelangt, folgendermaassen ausdrucken: Der 
Aragonil besteht aus einem Aggregat von monoklinen, gleich orientirten Theilchen, die nach einem rhombischen Skelett angeordnet sind. Aus dieser Anordnung der Molekelhaufen geht natürlich hervor, dass die schiefe und geneigte Dispersion der optischen Axen Null sein muss.

Die Symmetrieebene des Aragonits ist parallel zur Fläche (010), und die Symmetrieaxe parallel zur Kante [010].

Darnach besteht der Aragonit von Sicilien aus folgenden Krystallformen :

$S_{02}^{\sigma}\{010\},\{110\},\{111\},\{111\},\{021\},\{231\},\{231\},\{001\}$. Fig. 13.

Die Ebene der optischen Axen ist senkrecht zur Symmetrieebene.

Die stumpfe Bisetrix (positiv) $c$ fällt mit der Symmetrieaxe zusammen.

$$
\begin{aligned}
& 2 V=-18^{\circ} . \\
& \varrho>v \text { schwach, } \\
& G=2,925 .
\end{aligned}
$$

Spaltung parallel der Symmetrieebene deutlich; die zu $S_{02}^{\sigma}(110)$ und $S_{02}^{\sigma}(011)$ parallelen Spallungen sind kaum bemerk bar. 